\title{
Estimation of Dynamic Behaviors of Bedrock Foundation Subjected to Seismic Loads Based on FEM and DEM Simulations
}

\author{
Lei Yang*, Yujing Jiang**, Bo Li***, Shucai Li****, and Gang Wang ${ }^{* * * * *}$ \\ *Ph.D. Student, Graduate School of Science and Technology, Nagasaki University, Nagasaki \\ 852-8521, Japan, and Research Center of Geotechnical and Structural Engineering, Shandong \\ University, Jinan 250061, China (Corresponding Author, E-mail: sdylei@gmail.com) \\ **Professor, Faculty of Engineering, Nagasaki University, Nagasaki 852-8521, Japan, and Key \\ Laboratory of Mine Disaster Prevention and Control, Shandong University of Science and \\ Technology, Qingdao 266510, China (E-mail: jiang@nagasaki-u.ac.jp) \\ ***Assistant Professor, Faculty of Engineering, Nagasaki University, Nagasaki 852-8521, Japan \\ (E-mail: libo@nagasaki-u.ac.jp) \\ ****Professor, Research Center of Geotechnical and Structural Engineering, Shandong University, \\ Jinan 250061, China (E-mail: lishucai@sdu.edu.cn) \\ *****Associate Professor, Key Laboratory of Mine Disaster Prevention and Control, Shandong \\ University of Science and Technology, Qingdao 266510, China (E-mail: \\ Wanggang1110@gmail.com)
}

Abstract: Seismic behavior of bedrock foundation remains as one of the most fundamental and important problems concerning the stability and safety of nuclear power plants. The dynamic FEM (Finite Element Method) is commonly utilized in analyzing the seismic responses of bedrock; while in recent decades, the DEM (Distinct Element Method) has attracted more and more attentions, which has a better capability of simulating the sliding and separation of discontinuities in dynamic simulations. In this study, the FEM and DEM were adopted to investigate the seismic behavior of the bedrock of a nuclear power plant located in Japan, and the differences between the two methods in dynamic simulations were illuminated. Simulation results using FEM and DEM models without discontinuities agree well with each other, exhibiting an amplification effect of intact bedrock on the seismic wave propagation. Numerical simulation results obtained from the models containing faults give similar responses of bedrock to the input seismic waves; however, the FEM model underestimates the weakening effect of discontinuities on the propagation of seismic waves due to that it cannot represent well the large deformational behavior of discontinuities. When large deformation happens due to large seismic loads, the DEM can be regarded as a better method in seismic response evaluations of bedrock with discontinuities.

Keywords: seismic behavior, earthquake, bedrock foundation, numerical simulation, finite element method (FEM), distinct element method (DEM). 


\section{Introduction}

The world is now facing the combined challenge of dwindling fossil fuels and mounting energy demand. As an efficient and clean energy, nuclear energy has been developed and adopted by an increasing number of countries to secure their energy supplies and to protect the environment. Up to the end of 2010, 441 nuclear reactors distributed in 30 countries are in operation, producing $13.5 \%$ of the total electricity generation (International Atomic Energy Agency, 2011).

In the development of nuclear power industry, the seismic resistance performance of nuclear power plants is regarded as one of the key issues from the academic and engineering aspects. Due to the fact that nuclear power plants are energy facilities with potential danger, they have higher seismic-resistant requirements comparing with common industry and civil buildings (Qi and Qian, 2000). High-magnitude earthquakes can cause shutdown of nuclear plants, even leading to nuclear leakage, which would bring great harm to the environment and human society. For example, the M7.6 Jiji earthquake happened in Taiwan in 1999 caused the shutdown of three nuclear plants; the M6.8 Chuetsu offshore earthquake happened in Niigata, Japan in 2007 resulted in the leakage of radioactive material from the Kashiwazaki-Kariwa nuclear power plant, which is located $21 \mathrm{~km}$ away from the epicenter; and the M9.0 Great Tohoku Earthquake happened in Japan in 2011 also led to the leakage of radioactive material in the Fukushima Daiichi nuclear power plant.

Many countries and organizations have enacted corresponding regulations and guidelines to the site selection, stability estimation of bedrock foundation and seismic design of nuclear power plants (China Earthquake Administration, 1998; International Atomic Energy Agency, 2003; Nuclear Standards Committee of Japan, 2008a and 2008b). To improve the seismic resistance performance, most of the nuclear power plants have been built on bedrock. Therefore, the responses of bedrock foundation to seismic loads affect the safety of nuclear power plants to a great extent. Moreover, there are a mass of discontinuities like joints and faults existing in the bedrock, with various sizes and shapes, decreasing the strength of bedrock to some extent. Since rock masses with discontinuities are much weaker than the intact rock, the effect of discontinuities on the seismic behavior of bedrock is a key issue when assessing the total response of bedrock to seismic loads.

The research on the seismic responses of bedrock initiated from the simplified model of uniform horizontal soil layers with the seismic waves propagating vertically to the ground surface, which can be considered as a 1-D problem (Shou et al., 1982; Japan Society of Civil Engineers, 1989). Schnabel et al. (1972), Seed et al. (1986) and Idriss et al. (1974) performed a series of fundamental studies on the seismic response of ground, which pioneered the new field of the geotechnical earthquake engineering. In recent years, a number of theoretical and numerical researches on the seismic responses of foundations have been carried out, focusing on some more complex problems. Kinoshita (1984) proposed two theoretical models to predict the velocity response spectrums of 
bedrock. When the seismic period exceeds 1 second, these models agree well with the measured data. By using statistical methods, Kamiyama and Yanagisawa (1986) studied the influences of hypocenter characteristics, propagation path of seismic waves and mechanical properties of ground on the seismic responses of the ground foundation. Ushiyama et al. (2009) investigated the seismic characteristics of the Rokko fault rock in Kansi through FEM simulations. Zhao et al. (1988) established a coupling model of finite and infinite element to investigate the influences of soil properties (the natural vibration period and damping) and wave characteristics on the seismic response of foundation. Chen et al. (2006) investigated the effects of artificial boundary condition, model extent and damping factor on the seismic response using FEM, and developed the FEM code of SR2D. You et al. (2009) performed researches on the seismic responses of uniform horizontal soil layers with oblique incident waves, reporting that the incident angle has an obvious effect on the peak values of responding acceleration waves. Lü et al. (2010) adopted the explicit dynamic finite difference method to investigate the effect of hardness degree of bedrock on the foundation's seismic responses and acceleration response spectrum.

At present, the seismic behavior of bedrock foundations, especially the influence of discontinuities on the seismic responses of bedrock, are still not well understood. The FEM (Finite Element Method) is commonly adopted in most of the previous studies, taking into account the seismic response of foundations (Idriss et al., 1974; Zhao et al., 1988; Chen et al., 2006; Ushiyama et al., 2009) and underground \& surface structures (Jiang et al., 2010; Ozdemir et al., 2010; Clouteau et al., 2012). As a method of continuum mechanics, the FEM can not well represent the large deformation of discontinuities in rocks, which is an important factor in the propagation of seismic waves. In recent years, the DEM (Distinct Element Method) attracts more and more attention, due to its capability in simulating large deformations like sliding and separation of discontinuities (Jiang et al., 2009; Li et al., 2010) as well as the non-linear dynamic calculation (Zhang et al., 1997). The DEM simulations of seismic wave propagation across normally-distributed single or multiple rock fractures have been carried out by Cai and Zhao (2000) and Zhao et al. (2006 and 2008) by using the code of UDEC, and those simulation results, including the reflection \& transmission coefficients and the influence of joint stiffness on wave propagation, agreed well with the theoretical values proposed by Pyrak-Nolte et al. (1990) and Schoenberg (1980), verifying the validity and accuracy of DEM in seismic simulations.

In this study, both the FEM and the DEM were adopted to investigate the seismic behavior of the bedrock foundation under a nuclear power plant located in Japan. Through the comparison of numerical results, the influence of discontinuities on the seismic responses of bedrock was investigated and the differences between the two methods in seismic simulations of bedrock were clarified. 
Table 1. Physico-mechanical properties of intact rock and discontinuity

\begin{tabular}{ccccc}
\hline & Parameters & Indices & Units & Values \\
\hline \multirow{4}{*}{ Rock } & Density & $\rho$ & $\mathrm{g} / \mathrm{cm}^{3}$ & 2.66 \\
& Elastic modulus & $E$ & $\mathrm{GPa}$ & 71 \\
& Poisson's ratio & $v$ & - & 0.18 \\
& Tensile strength & $\sigma_{t}$ & $\mathrm{MPa}$ & 10.2 \\
& Cohesion & $c_{r}$ & $\mathrm{MPa}$ & 22.3 \\
& Friction angle & $\varphi_{r}$ & $\circ$ & 62 \\
\hline \multirow{4}{*}{ Discontinuity } & Normal stiffness & $k_{n}$ & $\mathrm{MPa} / \mathrm{mm}$ & 31.78 \\
& Shear stiffness & $k_{s}$ & $\mathrm{MPa} / \mathrm{mm}$ & 3.22 \\
& Cohesion & $c_{d}$ & $\mathrm{MPa}$ & 0.027 \\
& Friction angle & $\varphi_{d}$ & $\circ$ & 35.9 \\
\hline
\end{tabular}

\section{Characteristics of Bedrock and Seismic Wave}

\subsection{Properties of Bedrock}

The bedrock of the objective nuclear plant belongs to Mesozoic cumulates (conglomerate stone). The in-situ survey indicates that there are lots of discontinuities existing in the bedrock, among which three major faults exist, with the dip angles of $72^{\circ}, 56^{\circ}$ and $63^{\circ}$, respectively, having a controlling effect on the mechanical and deformational behavior of the bedrock.

To obtain the mechanical properties of intact rock, uniaxial and triaxial compression tests on intact rock samples taken from the objective ground (cylindrical samples with the size of diameter $\times$ height $=$ $50 \times 100 \mathrm{~mm}$ ) were carried out to measure the elastic modulus $(E)$, the Poisson's ratio $(v)$, the cohesion $\left(c_{r}\right)$ and the friction angle $\left(\varphi_{r}\right)$ of the rock matrix, and Brazilian tests on intact samples (diameter $\times$ height $=50 \times 25 \mathrm{~mm}$ ) were performed to measure the tensile strength $\left(\sigma_{t}\right)$ of intact rock. Comparing with the rock matrix, the pre-existing discontinuities have a more significant influence on the macro-mechanical behavior of bedrock. The mechanical properties of discontinuities were estimated by using the test approaches reported by Li et al. (2010) and Jiang et al. (2004). Normal loading tests were carried out on fractured rock samples (width $\times$ height $\times$ length $=100 \times 100 \times 200 \mathrm{~mm}$ ) with and without single natural discontinuities to acquire the total normal displacement $u_{\mathrm{t}}$ and the intact normal displacement $u_{\mathrm{i}}$ respectively. Then the normal displacement of the discontinuity can be derived from $u_{\mathrm{d}}$ $=u_{\mathrm{t}}-u_{\mathrm{i}}$ and the normal stiffness $\left(k_{n}\right)$ of discontinuity can be obtained from the normal displacement-normal stress curves. Direct shear tests on fractured rock samples were performed and the slope of the shear displacement-shear stress curves represents the shear stiffness $\left(k_{s}\right)$ of the discontinuity. The friction angle $\left(\varphi_{d}\right)$ and cohesion $\left(c_{d}\right)$ of the discontinuity were obtained from the normal stress-shear stress curves by connecting the peak shear stresses at different normal stresses. The physico-mechancial properties of the intact rock and discontinuity obtained from the above tests are 


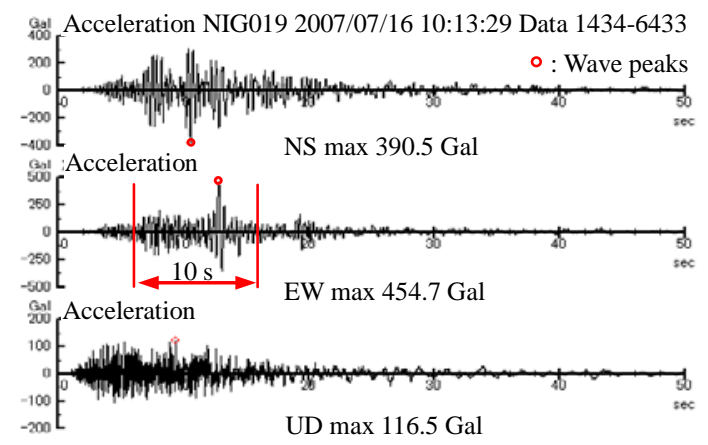

Fig. 1. Acceleration waves obtained at the Ojiya observation site during the Chuetsu offshore earthquake in Niigata (July 16, 2007)

shown in Table 1. As can be seen in the table, the bedrock is very strong, therefore, the major faults may present obvious influence on the propagation of the seismic waves, thus affect the seismic behavior of the bedrock.

\subsection{Seismic Wave}

Comparing with artificial waves, the real seismic waves taken from similar sites will be more representative to the situation when earthquake happens. In this study, the seismic wave obtained from the Ojiya observation site during the M6.8 Chuetsu offshore earthquake happened on July 16, 2007 in Niigata Prefecture, Japan was selected as the input wave. Fig. 1 shows the acceleration wave measured at the Ojiya observation site, which consists of three components along the NS, EW and UD directions respectively. Due to the fact that the damage to the bedrock and buildings caused by transverse waves (S-waves) is more severe than that caused by longitudinal waves (P-waves), the EW component of the S-wave with a larger seismic amplitude was selected as the input seismic wave in this study.

\section{Simulation of Seismic Wave at Deep Rock Formation}

The seismic waves obtained from observation sites during an earthquake represent the seismic motion of the ground surface. During earthquakes, seismic waves propagate from the deep rock formation to the ground surface. To reproduce such a process in numerical simulations, the seismic waves at the deep rock formation need to be obtained.

\subsection{Theory of Repeated Seismic Reflection}

The model of repeated seismic reflection assumes that rock layers distribute horizontally in a half space, constituted by homogenous, isotropic and viscoelastic rock medium. The origins of local 
coordinates are located at the upper surface of each layer, with the positive direction of the $X$-axis going downward perpendicularly. During the propagation of simple harmonic waves, the horizontal displacement of rock particles $u(x, t)$ induced by shear wave can be obtained by solving the wave equation, in the form of Eq. (1):

$$
u(x, t)=E e^{i(k x+\omega t)}+F e^{-i(k x-\omega t)}
$$

where $k$ is the wave number; $x$ is the coordinate value; $\omega$ is the angular frequency; $t$ is time; $E$ is the amplitude of incident wave; $F$ is the amplitude of reflected wave; and $i$ is the imaginary unit.

Then the velocity and acceleration of rock particles in terms of coordinate $x$ and time $t$ can be obtained by calculating the first and second partial derivatives of the displacement with respect to time. In addition, the shear stress $\tau(x, t)$ can also be derived from the displacement function based on the elastic theory, in the form of Eq. (2):

$$
\tau(x, t)=G \frac{\partial u}{\partial x}+\eta \frac{\partial^{2} u}{\partial x \partial t}
$$

where $G$ is the shear modulus and $\eta$ is the viscosity coefficient.

The compatibility conditions of displacement and shear stress on interfaces between adjacent layers need to be satisfied. Therefore the relation of amplitudes between any two adjacent layers can be derived in the following form:

$$
\left[\begin{array}{c}
E_{m+1} \\
F_{m+1}
\end{array}\right]=\frac{1}{2}\left[\begin{array}{ll}
\left(1+\alpha_{m}\right) e^{i k_{m} h_{m}} & \left(1-\alpha_{m}\right) e^{-i k_{m} h_{m}} \\
\left(1-\alpha_{m}\right) e^{i k_{m} h_{m}} & \left(1+\alpha_{m}\right) e^{-i k_{m} h_{m}}
\end{array}\right]\left[\begin{array}{c}
E_{m} \\
F_{m}
\end{array}\right]
$$

where $\alpha_{\mathrm{m}}$ represents the impedance and $m$ is the layer number.

Equation (3) indicates that the seismic amplitudes of any layer can be derived from those of their adjacent layers. Therefore, if the amplitudes of the first layer (ground surface) is known ( $E_{1}=F_{1}$, due to the fact that the shear stress on the ground surface is zero), all the amplitudes in different layers can be derived, and then the displacement function can be solved.

The equations mentioned above are only suitable for the cases of harmonic waves. Although the real seismic waves are much more complicated, they can be translated into the superposition forms of multiple harmonic waves through the Fourier transform. Seismic waves after the Fourier transform are in the form of frequency domain, which can be translated back into the time-domain forms through the inverse Fourier transform. 


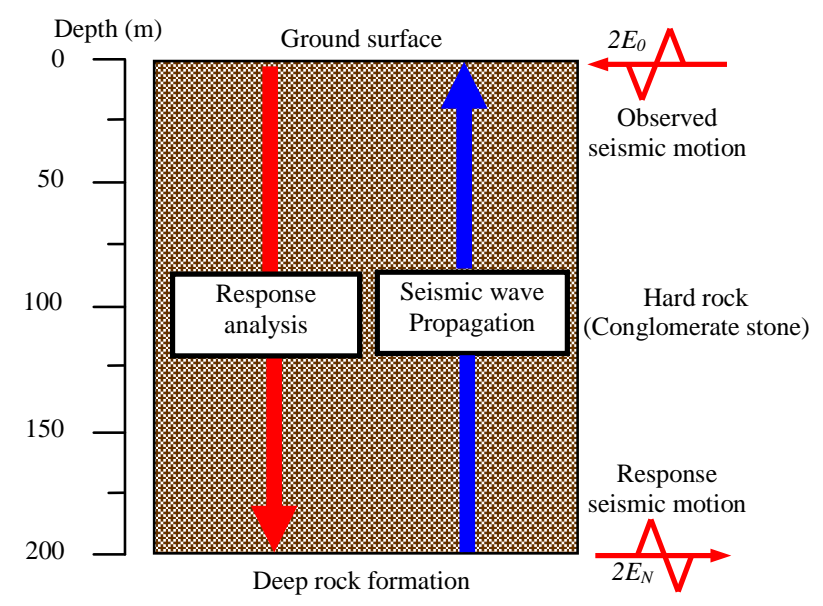

Fig. 2. Numerical model for investigating the seismic wave at the deep rock formation with a depth of $200 \mathrm{~m}$

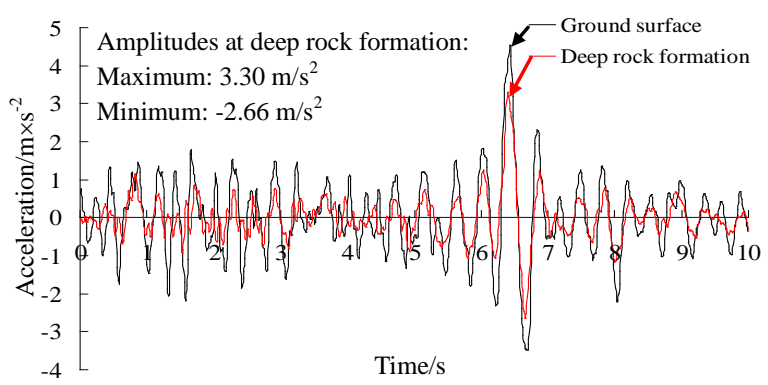

(a)

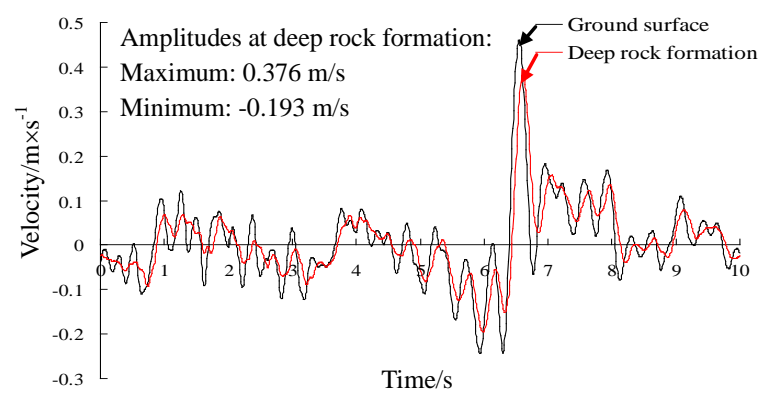

(b)

Fig. 3. Seismic waves on the ground surface and at the deep rock formation with a depth of $200 \mathrm{~m}$ : (a) Acceleration wave, (b) Velocity wave

\subsection{Input Seismic Wave at Deep Rock Formation}

The code of $k$-SHAKE developed by the Structure and Planning Institute of Japan (2005a) was used to calculate the seismic motion at the deep rock formation under the objective nuclear power 
plant, as shown in Fig. 2. The rock formation as deep as $200 \mathrm{~m}$ was chosen as the boundary of numerical model, on which the velocity and acceleration waves were calculated by using the theory of repeated seismic reflection mentioned above. During the calculation, the propagation velocity of S-wave in this bedrock was set as $3363 \mathrm{~m} / \mathrm{s}$, and the damping ratio was set as $3 \%$, according to the results of in-situ tests.

Dynamic simulations are very time consuming, therefore, in this study, only parts of the acceleration and velocity waves, which last 10 seconds (6.8-16.8 s, see Fig. 1) and contain the peak amplitudes of the total waves, were selected as the input waves to the DEM and FEM models, respectively, as shown in Fig. 3. The time-domain seismic waves were transformed into frequency-domain waves by the Fast Fourier Transform (FFT) to investigate the frequency characteristics. The FFT results indicate that the seismic waves present low-frequency characteristics with the dominant frequency of $2.3 \mathrm{~Hz}$ for the acceleration wave and $0.4 \mathrm{~Hz}$ for the velocity wave. In the waves, the time gap of the peak values between the seismic waves on the ground surface and at the deep rock formation ( $200 \mathrm{~m}$ ) can be observed, which is caused by the wave propagation. The peak values on the ground surface are larger than those at the deep rock formation, due to the amplification effect of bedrock.

\section{Numerical Simulations Based on FEM and DEM}

\subsection{Numerical Models of FEM and DEM}

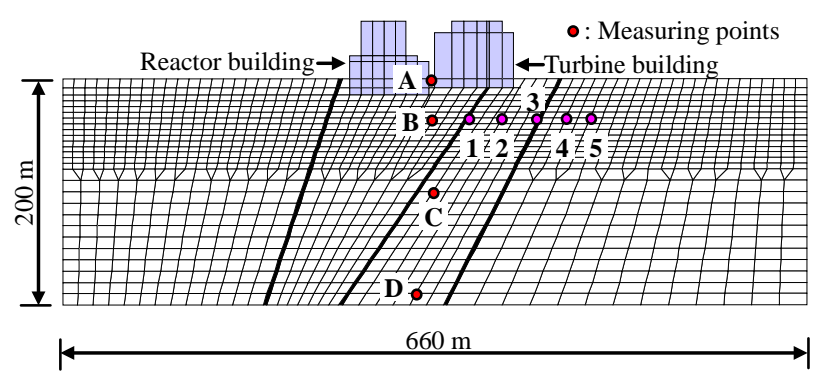

(a)

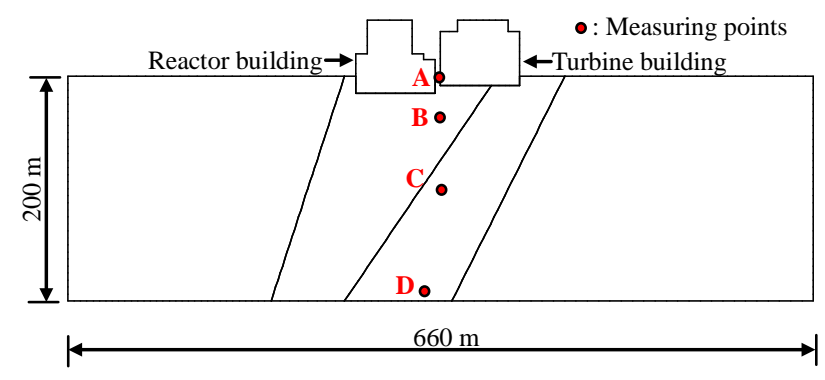

(b)

Fig. 4. Numerical simulation models: (a) FEM model, (b) DEM model 
Numerical simulations on the seismic behavior of the bedrock foundation were carried out by using the FEM code of SuperFLUSH/2D developed by the Structure and Planning Institute of Japan (2005b) and the DEM code of UDEC developed by the Itasca Consulting Group Inc. (2004).

According to the dimension of the nuclear facilities (the reactor and turbine buildings), the size of the numerical model was determined as $200 \mathrm{~m} \times 660 \mathrm{~m}$ (height $\times$ width) to sufficiently represent the deformational behavior and stress distribution in the bedrock. Three major faults mentioned above were set in the numerical models. The FEM and DEM models were established with the same dimension and boundary conditions, as shown in Fig. 4. To clarify the influence of discontinuities, the FEM and DEM models without faults were also established for the purpose of comparison. To investigate the seismic responses of bedrock at different locations, 4 monitoring points were set in the middles of the FEM and DEM models, marked as points A, B, C and D with the depths of $0 \mathrm{~m}$, $30 \mathrm{~m}, 100 \mathrm{~m}$ and $190 \mathrm{~m}$, respectively.

In the FEM model, plane strain elements and joint elements were used to represent the intact rock and faults respectively. In the DEM model, the Mohr-Coulomb elements and Coulomb joint elements were used for the intact rock and faults respectively. Triangular meshes (zones) with the side length of $10 \mathrm{~m}$ were generated in the DEM model to simulate the internal deformation.

The upwards-propagating seismic wave was input from the bottoms of models. On the bottom of each model, the identical viscous boundary was assigned to absorb the wave energy dissipating from the interior model. The viscous boundary employs a number of independent dashpots in the normal and shear directions, which can be effective at absorbing body and surface waves approaching the boundary. Lateral boundaries were set as free semi-infinite regions, which ensure the propagation of seismic waves out of the model. In the DEM model, the lateral free-field boundaries were adopted to enhance the free-field motion of model boundaries and to absorb the outward waves by using viscous dashpots (Itasca Consulting Group Inc., 2004). In the FEM model, the lateral energy-transmission boundaries were adopted to absorb waves by calculating the boundary matrix of energy transmission, which can be obtained via the Eigen analysis of the motion equation (Structure and Planning Institute of Japan, 2005b).

\subsection{Calculation Principles of FEM and DEM}

In FEM simulations, the equation of motion can be written in the discretization form of Eq. (4):

$$
[M]\{\ddot{u}\}+[C]\{\dot{u}\}+[K]\{u\}=-[M]\{1\} \ddot{u}_{b}
$$

where $[M]$ is the mass matrix; $[C]$ is the damping matrix; $[K]$ is the stiffness matrix; $\{\ddot{u}\}$ is the acceleration vector; $\{\dot{u}\}$ is the velocity vector; $\{u\}$ is the displacement vector; $\{1\}$ is the unit vector; and $\ddot{u}_{b}$ is the input seismic acceleration. The deformations of intact rock and discontinuities in the FEM model are involved in the same matrixes. The FEM code of SuperFLUSH/2D employs an 
implicit integration method during the dynamic simulation, which can be effective to reduce the running time.

In DEM simulations, the fractured rock mass is represented by an assemblage of blocks and interfaces, which model the rock matrix and discontinuities respectively. The motion equation of a block can be written in the form of central difference scheme:

$$
\begin{aligned}
& \dot{u}_{i}^{(t+\Delta t / 2)}=\dot{u}_{i}^{(t-\Delta t / 2)}+\left(\frac{\Sigma F_{i}^{(t)}}{m}+g_{i}\right) \Delta t \\
& \dot{\theta}^{(t+\Delta t / 2)}=\dot{\theta}^{(t-\Delta t / 2)}+\left(\frac{\Sigma M_{i}^{(t)}}{I}\right) \Delta t
\end{aligned}
$$

where $t$ is time; $\Delta t$ is time step; $u_{i}$ is the displacement component of block centroid; $\sum F_{i}$ is the total external force; $m$ is mass; $g_{i}$ is the gravitational acceleration; $\theta$ is the angular displacement; $\sum M_{i}$ is the total moment acting on the block; and $I$ is the moment of inertia of block. The symbol '. ' above letters denotes the partial derivative with respect to time. Eq. (5) shows that the dynamic behavior within a DEM simulation is represented numerically by a time-stepping algorithm, with the advantage that it operates in the time domain.

The DEM treats the dynamic behavior of fractured rock mass in a more effective way. An explicit integration method is employed to compute the change in the position and velocity of each element during a certain time step from the Newton's law of motion. Then the new positions and velocities are used to compute the forces at the interfaces and in the rock matrix during the next step based on the force-displacement law, and such loop is repeated until the simulation ends (Itasca Consulting Group Inc., 2004). The explicit integration method requires the timing step in DEM simulations to be small enough for the purpose of numerical stability, as a result, the running time of the DEM simulation in this study is approximately two times of that of the implicit FEM simulation (with the same CPU and memory).

Damping is an important factor in dynamic simulations, due to the energy loss as a result of internal friction in the intact material and deformations (seperation and sliding) along interfaces within the system. The Rayleigh damping was used in both FEM and DEM models with the form of Eq. (6). The coefficients $\alpha$ and $\beta$ stand for the mass-proportional and stiffness-proportional damping coefficients respectively, which are related to the dominant frequency of seismic waves (in this study, $f_{d}=2.3$ or $0.4 \mathrm{~Hz}$ for the acceleration or velocity wave) and the damping ratio of bedrock ( $\xi_{\text {rock }}=$ $3 \%)$, in the form of Eq. (7).

$$
[C]=\alpha[M]+\beta[K]
$$




$$
\alpha=2 \pi \xi_{\text {rock }} f_{d}, \quad \beta=\frac{\xi_{\text {rock }}}{2 \pi f_{d}}
$$

The basic deference between FEM and DEM focuses on the treatment of discontinuities existing in rock masses. At present, the treatment of discontinuities remains the most important limiting factor in the application of the FEM for rock mechanics problems (Jing and Hudson, 2002). Although some representation methods for discontinuities like the joint element have been widely implemented in FEM codes, these models are formulated based on continuum assumptions assuming that the displacements are continuous across the discontinuities, and the large-scale deformations like opening and sliding of discontinuities are not permitted. In the DEM, the discontinuities are treated as interfaces between rock blocks. The interface can be regarded as a kind of boundary condition, which is capable of simulating the large-deformational behavior of discontinuities. Therefore, the important feature of displacement discontinuity for rock discontinuities, which has significant influence on the propagation of seismic waves, can be well represented. Moreover, in the DEM (UDEC) simulations, the wave scattering when seismic waves travel across discontinuities is taken into account, by using the coefficients of reflection $(R)$, transmission $(T)$ and absorption $(A)$, in the forms of Eq. (8):

$$
R=\sqrt{\frac{E_{R}}{E_{I}}}, \quad T=\sqrt{\frac{E_{T}}{E_{I}}}, \quad A=\sqrt{1-R^{2}-T^{2}}
$$

where $E_{I}, E_{T}$ and $E_{R}$ denote the energy flux per unit area per cycle of oscillation associated with the incident, transmitted and reflected waves, respectively.

\subsection{Simulation Results}

The horizontal velocity waves measured at different monitoring points in the FEM and DEM models without faults are shown in Fig. 5. The response seismic waves obtained from those two methods show good consistency in the aspects of waveforms and amplitudes (the differences of peak values are less than $3.36 \%$, with the average value of $1.68 \%$ for four monitoring points, which may be caused by the different time integration methods adopted in the FEM and DEM). In the simulation results, the wave amplitudes increase during the propagation of seismic waves from the deep rock formation to the ground surface (see Fig. 6), which is known as an amplification effect of the intact bedrock. These results show that in seismic simulations of intact bedrock (without discontinuities), the numerical results of the FEM and DEM models agree well with each other.

Fig. 7 shows the horizontal velocity waves measured at four monitoring points with varying depths in the FEM and DEM models containing three major faults (Fig. 4). In the FEM model (Fig. 7(a)), the response waveforms at different monitoring points are consistent despite of the difference in peak values (maximum amplitudes) at different depths. As the seismic waves propagate from the 
deep rock formation to the ground surface, the amplitudes of waves increase as can be observed at peaks and troughs, just like the case of intact bedrock. The bedrock has an amplification effect on the amplitudes of the seismic waves in the FEM model. Since the seismic wave travels only about $0.06 \mathrm{~s}$ through this bedrock, no obvious delay was observed within the response waves at different monitoring points. In the DEM simulation results (Fig.7(b)), although the velocity waves at different locations have similar waveforms, the response waves show different characteristics from the FEM simulation results, mainly in two aspects: the amplitude variation at peaks and troughs of waves is more obvious; the peaks and troughs of waves appear at different times in different locations. In addition, with the upward propagation of seismic waves, the amplitudes of response waves decrease, which are opposite to that observed in the FEM simulations.

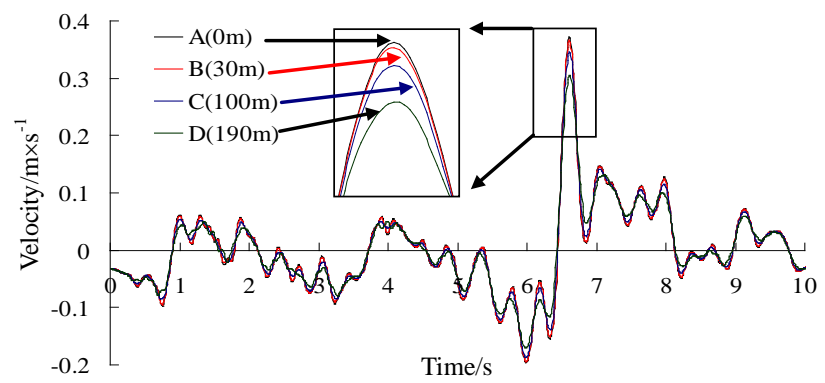

(a)

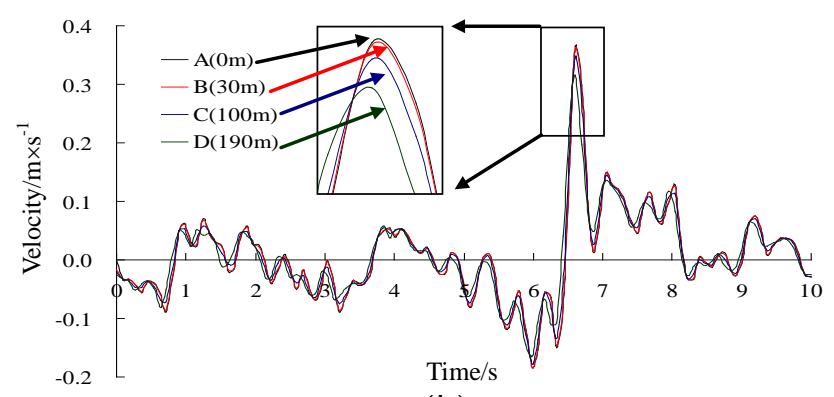

(b)

Fig. 5. Horizontal velocity waves measured at different monitoring points in FEM and DEM models without faults: (a) FEM model, (b) DEM model

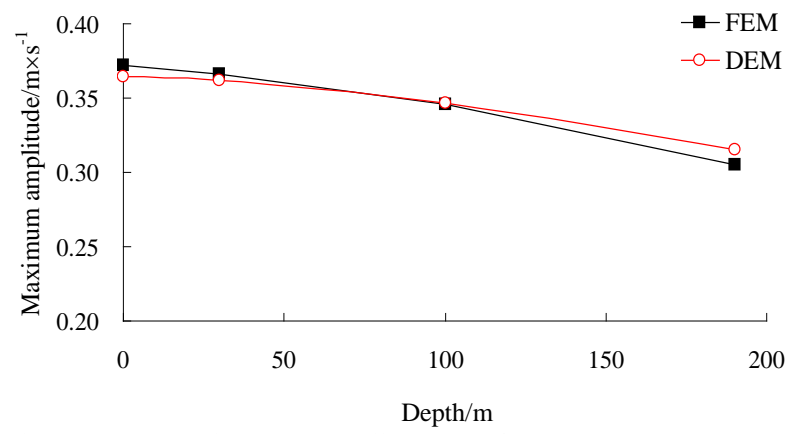

Fig. 6. Relationship between maximum amplitudes of response waves and depths within FEM and DEM models without faults 


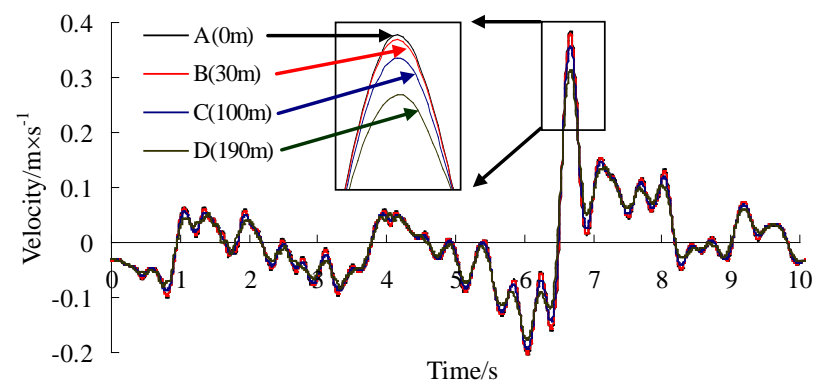

(a)

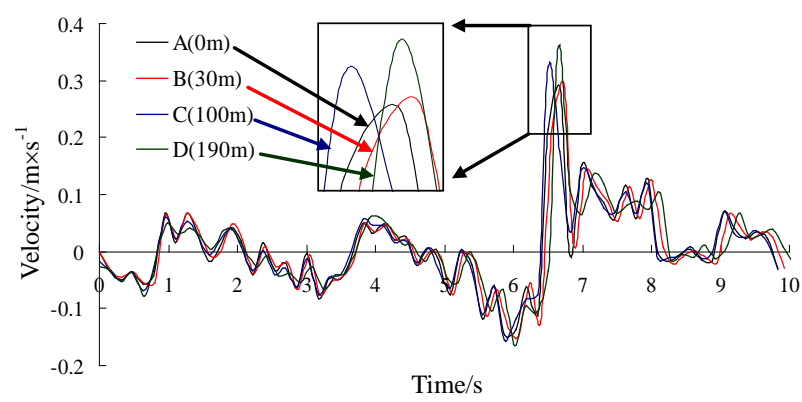

(b)

Fig. 7. Horizontal velocity waves measured at different monitoring points in FEM and DEM models with faults: (a) FEM model, (b) DEM model

\subsection{Comparisons of FEM and DEM Simulation Results}

The simulation results of the FEM and DEM models without faults agree well with each other, while the FEM and DEM models containing faults present different characteristics, in terms of waveform divergence and amplitudes variation at different depths. Those differences may result from the treatment of discontinuities in the two methods. When seismic waves propagate through discontinuities, scattering phenomena including reflection, refraction, transmission and diffraction occur, resulting in energy dissipation. In the FEM model, although the physico-mechanical parameters of faults were assigned on joint elements, large deformation such as sliding and separation of faults can not be adequately represented due to the assumption of continuous displacement across the discontinuities. Therefore, the FEM model may underestimate the faults' attenuation effect on the propagation of seismic waves in the aspects of velocity and energy, which can be found in the simulation results as shown in Figs. 5(a) and 7(a), where no obvious differences can be observed in the models with and without faults. To perform a further investigation into the faults' influence on seismic responses of bedrock in the FEM simulation, five monitoring points marked as 1, 2, 3, 4 and 5 were set in the FEM model, at the same depth of $30 \mathrm{~m}$ but in different positions (see Fig. 4(a)). Fig. 8 shows the response velocity waves at those monitoring points. All of these response waves are almost identical in waveforms and amplitudes, indicating that the existence 
of faults has little impact on the dynamic behavior of bedrock.

As a method based on discontinuum mechanics, the DEM treats the discontinuities as interfaces between blocks, thus has a better performance on presenting the deformational behavior of discontinuities, especially when large deformation happens like separation and sliding. Within the DEM simulations, the faults in bedrock behave as displacement discontinuities, resulting in the scatting of seismic waves and the dissipation of seismic energy, leading to the decrease of wave amplitudes during the wave propagation. In addition, the faults also result in divergence of waveforms at different locations due to the reflection, transmission and superposition of seismic waves (see Fig. 7(b)).

To make a further comparison of the FEM and DEM results, the response waves at depths of $0 \mathrm{~m}$, $30 \mathrm{~m}$ and $100 \mathrm{~m}$ were compared, as shown in Fig. 9. Those results show that although the waveforms obtained from these two methods are similar, the amplitudes of response waves from the DEM simulations are smaller than those from the FEM simulations, due to that the faults exhibit an attenuation (weakening) effect on the propagation of seismic waves, which were well represented in the DEM model but not in FEM model. In addition, the difference in wave amplitudes between FEM and DEM results becomes more obvious as the depth of the monitoring point becomes shallow (As the propagation distance increases, the attenuation effect of faults on seismic waves increases).

\section{Conclusions and Discussions}

In this study, both FEM and DEM dynamic simulations were conducted to investigate the seismic responses of bedrock of a nuclear power plant located in Japan to the seismic waves obtained from the Ojiya observation site during the Chuetsu offshore earthquake happened in Niigata, Japan. The code of k-SHAKE based on the theory of repeated seismic reflection was used to obtain the seismic wave at the deep rock formation with a depth of $200 \mathrm{~m}$, which was set as the bottom boundary of numerical models. The difference between the FEM and DEM in seismic simulations was investigated.

The seismic simulation results of FEM and DEM models without discontiniuties agree well with eath other in respects of waveforms and wave amplitudes, exhibiting an amplification effect of intact bedrock on the seismic wave propagation. The results of FEM and DEM models containing faults show quite different characteristics. The response waves at different locations from FEM simulations exhibit good consistency. Along with the seismic waves propagating from the deep rock formation to the ground surface, the amplitudes at the peaks and troughs of waves increase. On the contrary, in the DEM simualtion results, the amplitudes of seismic waves decrease with the upward propagation of seismic waves. The comparison of the response waves at the same locations indicates that the wave amplitudes obtained from the DEM simulations are smaller than those from the FEM simulations. The difference in wave amplitudes becomes more obvious along with the decrease of 
the depth of monitoring points.

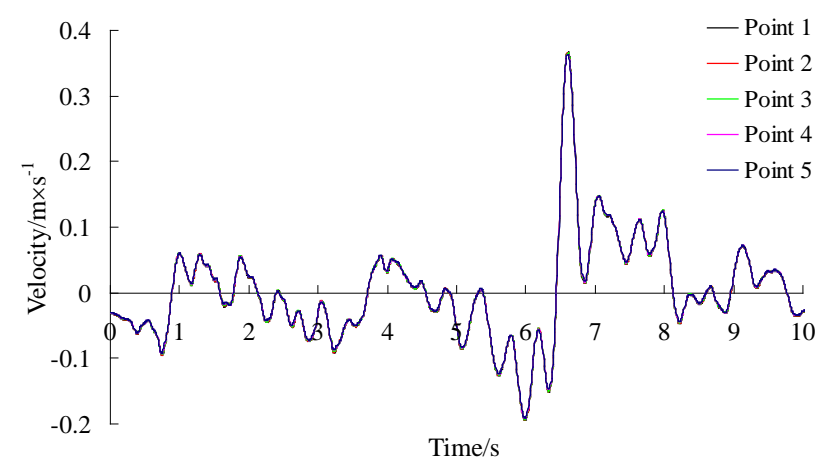

Fig. 8. Velocity waves at different monitoring points at the same depth in FEM model

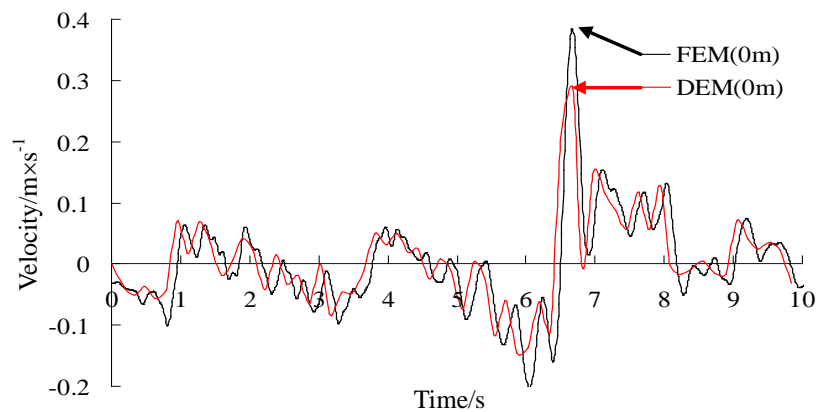

(a)

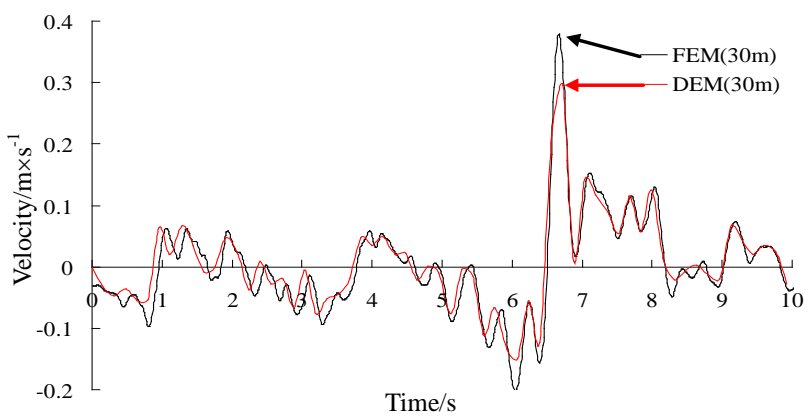

(b)

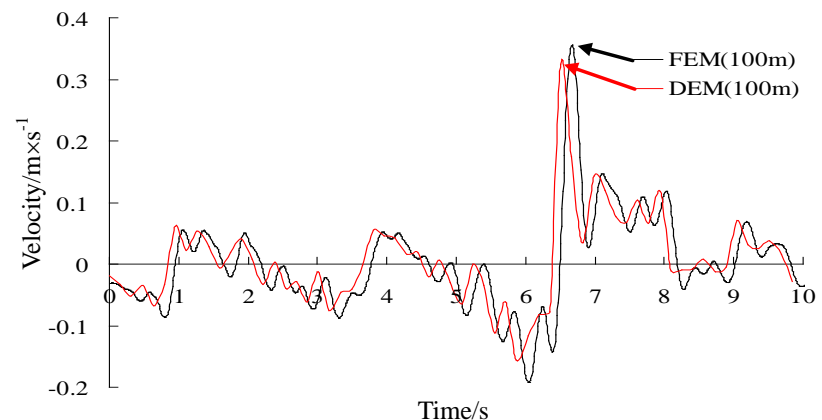

(c)

Fig. 9. Comparison of response waves at different locations obtained from FEM and DEM simulations: (a) Depth: 0 m, (b) Depth: 30 m, (c) Depth: 100 m 
The differences between FEM and DEM models are mostly resulted from the treatment of discontinuities. The FEM may in some contents underestimate the faults' attenuation effect on the propagation of seismic waves through a bedrock. Comparatively, the DEM treats the discontinuities in a more realistic way with the capability of simulating the large-deformational behavior of discontinuities, which help give better estimations to the seismic responses of bedrocks containing discontinuities. However, the DEM usually has a higher requirement for the computational power of computers than the FEM, and the maximum number of meshes (zones in rock blocks and contacts at interfaces) and the duration of dynamic simulations are limited by the computational power.

In this study, the 2-D FEM and DEM models were employed to investigate the seismic responses of bedrock foundation. The 2-D models are incapable of representing some 3-D features of the bedrock, such as the spatial distribution of discontinuities and the spatial anisotropy of material properties. Therefore, future researches will be carried out with 3-D FEM and DEM models, focusing on the influences of spatially-distributed discontinuities and their mechancial properties on the seismic responses of bedrock foundation, as well as the mechancial and failure behavior of bedrock foundation under seismic loads.

\section{Acknowledgment}

This work was financially supported by the Program for Changjiang Scholars and Innovative Research Team in University (No. IRT0843), the National Program on Key Basic Research Project (973 Program) (Grant No.2010CB226805) and the Research Award Fund for Outstanding Middle-aged and Young Scientist of Shandong Province of China (BS2010HZ015).

\section{References}

Cai, J. G. and Zhao, J. (2000). "Effects of multiple parallel fractures on apparent attenuation of stress waves in rock masses.” International Journal of Rock Mechanics \& Mining Sciences, Vol. 37, No. 4, pp. 661-682.

Chen, C., Lou, M., and Tao, S. (2006). "Discussions on two-dimensional finite element analysis of seismic response.” Technology for Earthquake Disaster Prevention, Vol. 1, No. 4, pp. 292-301.

China Earthquake Administration (1998). GB 50267-97 Code for seismic design of nuclear power plants, China Planning Press, Beijing, China.

Clouteau, D., Broc, D., Devésa, G., Guyonvarh, V., and Massin, P. (2012). "Calculation methods of Structure-Soil-Structure Interaction (3SI) for embedded buildings: Application to NUPEC tests.” Soil Dynamics and Earthquake Engineering, Vol. 32, No. 1, pp. 129-142.

Idriss, I. M., Seed, H. B., and Serff, N. (1974). "Seismic response by variable damping finite elements.” Journal of Geotechnical Engineering Division, Vol. 100, No. 1, pp. 1-13. 
International Atomic Energy Agency (2003). NS-G-1.6 Safety guide of seismic design and qualification for nuclear power plant, Vienna, Austria.

International Atomic Energy Agency (2011). Energy, electricity and nuclear power estimates for the period up to 2050, Vienna, Austria.

Itasca Consulting Group Inc. (2004). Universal distinct element code: theory and background, Minnesota, USA.

Japan Society of Civil Engineers (1989). Dynamic analysis and seismic design, Japan Gihodo Press, Tokyo, Japan.

Jiang, L., Chen, J., and Li, J. (2010). "Seismic response of underground utility tunnels: shaking table testing and FEM analysis.” Earthquake Engineering and Engineering Vibration, Vol. 9, No. 4, pp. 555-567.

Jiang, Y., Li, B., and Yamashita, Y. (2009). "Simulation of cracking near a large underground cavern in a discontinuous rock mass using the expanded distinct element method.” International Journal of Rock Mechanics \& Mining Sciences, Vol. 46, No. 1, pp. 97-106.

Jiang, Y., Xiao, J., Tanabashi, Y., and Mizokami, T. (2004). "Development of an automated servo-controlled direct shear apparatus applying a constant normal stiffness condition.” International Journal of Rock Mechanics \& Mining Sciences, Vol. 41, No. 2, pp. 275-286.

Jing, L. and Hudson, J. A. (2002). "Numerical methods in rock mechanics.” International Journal of Rock Mechanics \& Mining Sciences, Vol. 39, No. 4, pp. 409-427.

Kamiyama, M. and Yanagisawa, E. (1986). “A statistical model for estimating response spectra of strong earthquake ground motions with emphasis on local soil condition.” Soils and Foundations, Vol. 26, No. 2, pp. 16-32.

Kinoshita, S. (1984). "Spectral Characteristics of bedrock motion in the Tokyo metropolitan area." Proceeding of Japan Society of Civil Engineers, No. 344/I-1, pp. 51-56, Tokyo, Japan.

Li, B., Jiang, Y., Tanabashi, Y., and Yamashita, Y. (2010). "Behavior of large scale underground cavern located in jointed rock masses evaluated by using Distinct Element Method.” Soils and Foundations, Vol. 50, No. 5, pp. 609-621.

Lü, T., Yang, Q., Geng, X., Yang, L., Yang, L., and Li, H. (2010). "Study of influence of rock hardness on characteristic of seismic response of bedrock-foundation of nuclear power plants.” Rock and Soil Mechanics, Vol. 31, No. 4, pp. 1319-1325.

Nuclear Standards Committee of Japan (2008a). JEAC 4601-2008 Technical regulations for seismic design of nuclear power plant, Japan Electric Association Press, Tokyo, Japan.

Nuclear Standards Committee of Japan (2008b). JEAG 4601-2008 Technical guide for seismic design of nuclear power plant, Japan Electric Association Press, Tokyo, Japan.

Ozdemir, Z., Souli, M., and Fahjan, Y. M. (2010). “Application of nonlinear fluid_structure interaction methods to seismic analysis of anchored and unanchored tanks." Engineering 
Structures, Vol. 32, No. 2, pp. 409-423.

Pyrak-Nolte, L. J., Myer, L. R., and Cook, N. G. W. (1990). “Transmission of seismic waves across single natural fractures.” Journal of Geophysical Research, Vol. 95, No. B6, pp. 8617-8638.

Qi, C. and Qian, Q. (2000). “Overview of seismic research for nuclear power plant.” Earthquake Engineering and Engineering Vibration, Vol. 20, No. 3, pp. 76-86.

Schnabel, P. B., Lysmer, J., and Seed, H. B. (1972). SHAKE: A computer program for earthquake response analysis of horizontally layered sites, EERC University of California, Berkeley, USA.

Schoenberg, M. (1980). "Elastic wave behaviour across linear slip interfaces.” Journal of Acoustics Society of America, Vol. 68, No. 5, pp. 1516-1521.

Seed, H. B., Wong, R. T., Idriss, I. M., and Tokimatsu, K. (1986). "Moduli and damping factors for dynamic analyses of cohesionless soils.” Journal of Geotechnical Engineering, Vol. 112, No. 11, pp. 1016-1032.

Shou, P., Liu, C., and Zhu, J. (1982). Application of seismic waves in projects, Earthquake Press, Beijing, China.

Structure and Planning Institute of Japan (2005a). User manual of the seismic response analysis program for stratified soil layers: $k$-SHAKE, Tokyo, Japan.

Structure and Planning Institute of Japan (2005b). User manual of the two-dimensional dynamic interaction analysis program for ground and structure: SuperFLUSH/2D, Tokyo, Japan.

Ushiyama, N., Taniyama, H., and Tsunoda, F. (2009). "Concussional movement of the Rokko fault block in Kansai and its dynamic response analysis for seismic isolation block system.” Earth Science, Vol. 26, No. 6, pp. 343-354.

You, H., Zhao, F., and Rong, M. (2009). "Nonlinear seismic response of horizontal layered site due to inclined wave." Chinese Journal of Geotechnical Engineering, Vol. 31, No. 2, pp. 234-240.

Zhang, C., Pekau, O. A., Jin, F., and Wang, G. (1997). "Application of distinct element method in dynamic analysis of high rock slopes and blocky structure." Soil Dynamics and Seismic Engineering, Vol. 116, pp. 385-394.

Zhao, C., Zhang, C., Wang, G., and Zhang, G. (1988). "Effects of the characteristics of soil layers on field seismic response by using infinite element.” Hydro-science and Engineering, Vol. 4, No. 1, pp. 31-45.

Zhao, X. B., Zhao, J., Cai, J. G., and Hefny, A. M. (2008). "UDEC modeling on wave propagation across fractured rock masses.” Computers and Geotechnics, Vol. 35, No. 1, pp. 97-104.

Zhao, X. B., Zhao, J., Hefny, A. M., and Cai, J. G. (2006). "Normal transmission of S-wave across parallel fractures with Coulomb slip behavior.” Journal of Engineering Mechanics, Vol. 132, No. 6, pp. 651-650. 